Marly Marques da Cruz ${ }^{1}$

\title{
O sistema de informação de AIDS do Município do Rio de Janeiro: suas limitações e potencialidades enquanto instrumento da vigilância epidemiológica
}

Luciano Medeiros de Toledo 1

Elizabeth Moreira dos Santos 1

\author{
Strengths and limitations of the Rio de Janeiro \\ Municipal AIDS Information System as an \\ instrument for epidemiological surveillance
}

${ }^{1}$ Departamento de Endemias Samuel Pessoa, Escola Nacional de Saúde Pública, Fundação Oswaldo Cruz. Rua Leopoldo Bulhões 1480, Rio de Janeiro, $R J$ 21041-210, Brasil. marly@ensp.fiocruz.br toledo@ensp.fiocruz.br bmoreira@ensp.fiocruz.br

\begin{abstract}
This study analyzes the Rio de Janeiro Municipal AIDS Information System, specifically the system's strengths and limitations in the epidemic's current context, from a broader perspective of epidemiological surveillance. The objective was to provide a brief description of the system, considering its different components. The authors further analyzed records pertaining to the reporting and investigation of AIDS cases from 1985 to 1995 and an update of the database as a contribution to the system's quality. The results showed improvement in the consistency of the AIDS database, particularly for the following variables: occupation, neighborhood, school, and exposure category, although the study found concrete limitations in the system's design and structure for dealing with the complexity and heterogeneity of AIDS surveillance. The study thus identified the need to construct an efficient HIVIAIDS surveillance system, based on epidemiological indicators aimed at monitoring and evaluating changes in the epidemiological pattern and analysis of the impact on morbidity and mortality resulting from access to more efficient forms of prevention and new treatments.
\end{abstract}

Key words Information Systems; Acquired Immunodeficiency Syndrome; Epidemiologic Surveillance

Resumo O estudo faz uma reflexão acerca do sistema de informação de AIDS, dando visibilidade às potencialidades e limitações deste sistema no contexto atual da epidemia e numa perspectiva mais ampliada de vigilância epidemiológica. Buscou-se fazer uma descrição do sistema, considerando seus diversos componentes. Além disso, tratou-se do resultado referente ao levantamento nas fichas de notificação/investigação de AIDS no período de 1985 a 1995, e, em uma atualização da base de dados como contribuição para a qualidade do sistema. Os resultados mostraram melhora na consistência do banco de dados de AIDS, em particular das variáveis: ocupação, bairro, escolaridade e categoria de exposição, embora tenham sido identificadas limitações concretas na concepção e na estrutura do sistema para dar conta da complexidade e heterogeneidade da vigilância da AIDS. Daí ter sido apontada a necessidade de estruturação de um eficiente sistema de vigilância do HIVIAIDS, tomando-se como referência os indicadores epidemiológicos direcionados ao monitoramento e à avaliação mediante acompanhamento das mudanças no padrão epidemiológico, como da análise do impacto na morbi-mortalidade decorrente do acesso a formas mais eficientes de prevenção, aos novos tratamentos e terapêticas.

Palavras-chave Sistemas de Informação; Síndrome de Imunodeficiência Adquirida; Vigilância Epidemiológica 


\section{Introdução}

O presente artigo visa contribuir para uma reflexão acerca do sistema de informação de AIDS, procurando dar visibilidade às suas potencialidades e limitações num contexto mais amplo de vigilância da AIDS no Programa de Doenças Sexualmente Transmitidas e AIDS da Secretaria Municipal de Saúde do Rio de Janeiro (SMS-RJ). Tal discussão remete à revisão da lógica de estruturação do sistema, aos comprometimentos em termos de consistência e algumas sugestões de reestruturação dentro de uma perspectiva de inclusão da vigilância do HIV.

Esta análise foi motivada pela busca de respostas às inquietações propiciadas pela constatação de significativa perda de dados observada no Sistema de Informação Nacional de Agravos de Notificação (SINAN) de AIDS, interferindo diretamente na qualidade das informações resultantes da análise dessa base de dados. As perdas tornavam evidentes as fragilidades das informações obtidas com o processamento dos dados, comprometendo a possibilidade de uma caracterização mais detalhada, do ponto de vista epidemiológico, da epidemia de AIDS no Município do Rio de Janeiro.

No âmbito nacional, considerando-se os distintos níveis de atuação, tem-se trabalhado ou pelo menos se aproximado da definição de vigilância epidemiológica de AIDS, como " $a$ coleta sistemática de dados epidemiológicos sobre a ocorrência de casos de AIDS, de interesse para o planejamento, a implementação e o acompanhamento das atividades e programas de controle e prevenção da infecção pelo HIV e da AIDS" (SES-RJ, 1998:32). No entanto, no decorrer da discussão procurar-se-á trabalhar com este sistema de vigilância como parte de um processo que tem como eixo a informação-açãodecisão enquanto controle da epidemia, enquanto necessidade de ampliação das ações de vigilância, para que esta não continue sendo reduzida à mera e exclusiva tarefa de ordem técnico-burocrática, ou mesmo de domínio exclusivo dos epidemiologistas.

Os dados que alimentam o sistema de vigilância epidemiológica de AIDS são oriundos das notificações universais de casos confirmados pela Definição de Caso de AIDS adotado no país, utilizando o SINAN em rede nacional, em parceria com as secretarias estaduais de saúde (MS, 1999). Este sistema tem como objetivo o registro e o processamento dos dados sobre os agravos de notificação compulsória em todo o território nacional, a fim de fornecer informações para a análise do perfil de morbidade vigente (Ferreira \& Portela, 1999).
Os autores defendem o ponto de vista de que as informações geradas pelo sistema de vigilância de AIDS devem fazer parte de um processo dinâmico, variável em função das mudanças do perfil epidemiológico, dos resultados obtidos com as ações de controle e da disponibilidade de novos conhecimentos científicos e tecnológicos.

Enquanto sistema de informação, o SINAN/ AIDS atende a uma dada racionalidade e lógica de funcionamento, que, no caso específico, possui como eixo delineador a definição de caso. É notável a potencialidade das informações produzidas com conseqüente subutilização deste sistema. Não se deve ainda perder de vista os problemas referentes à possibilidade de implementação da vigilância do HIV, dos mecanismos de controle concernentes à fidedignidade dos dados, assim como à compatibilização das informações, visando à identificação de mudanças no quadro epidemiológico da doença.

Nesse sentido, as informações geradas pelo sistema devem, de fato, subsidiar as ações da vigilância, o planejamento das ações de prevenção, o monitoramento e a avaliação de processos visando ao controle e ao combate do HIV e da AIDS. Deve-se destacar que um sistema de vigilância universal do HIV compreende as diferentes dimensões da história natural da infecção. Mais do que estudos/atividades isoladas de controle, ele abrange diversas dimensões hierarquizadas em relação à história natural, à complexidade tecnológica das ações de diagnóstico, ao tratamento e ao controle. Por exemplo, a vigilância biológica (rede de laboratório de soroprevalência e sorotipagem); vigilância comportamental (estudos transversais na população em geral e em grupos específicos) e outras fontes de informação como, por exemplo, a vigilância de casos de AIDS, os dados de mortalidade e os dados de DST (UNAIDS / WHO, 2000).

À discussão corrente dos documentos de referida temática parece interessar acrescentar duas dimensões complementares: a farmacovigilância, que abrangeria estudos de resistência aos anti-retrovirais e efeitos colaterais destes últimos, e estudos de validação, monitoramento e readequação do próprio sistema de informação.

Entretanto, esta discussão não condiz com a lógica da vigilância vigente, cujo dilema está em se registrarem dados, produzir informações que reflitam eventos complexos à necessidade de utilização de registros simples, ágeis operacionalmente, bem como as dificuldades de construí-los, paralelamente à construção da própria doença em si. O momento atual da epi- 
demia traz à tona a necessidade de estruturação de um sistema capaz de superar o esgotamento da vigilância de AIDS, tendo em vista as mudanças ocorridas na evolução da epidemia nos últimos anos. A implementação de novas formas de tratamento, assim como a mudança no padrão epidemiológico da AIDS ressaltam um cenário que prima pela agilidade da informação e pela intervenção cada vez mais precoce.

Com base nessas idéias, são enfatizadas algumas lacunas do sistema de informação e a importância em se investir na qualidade do mesmo no nível local. Este estudo visa, sobretudo, chamar atenção para a relevância do sistema de vigilância de AIDS na produção de informações mais fidedignas que sirvam, verdadeiramente, para o aprimoramento de ações assistenciais e preventivas das organizações governamentais e não governamentais, da mesma forma que venha apontar caminhos com o intuito de ampliação das atividades de vigilância epidemiológica no monitoramento da infecção pelo HIV.

\section{A vigilância epidemiológica de AIDS no Município do Rio de Janeiro}

\section{Um breve histórico do Sistema \\ de Informação Nacional de Agravos de Notificação}

Como forma de obter um melhor detalhamento da lógica e estrutura do sistema de informação adotado no processamento dos dados de AIDS, priorizou-se a apresentação de um breve histórico do SINAN. Afinal, este é um sistema nacional que, num sentido mais amplo, não atende só às necessidades da notificação/investigação de AIDS, mas também engloba a notificação/investigação de outros agravos de notificação.

O SINAN foi criado em 1990 para tentar sanar as dificuldades do Sistema Nacional de Doenças Compulsórias (SNDC), procurando racionalizar o processo de coleta e transferência de dados relacionados às doenças e agravos de notificação compulsória, já tendo em vista o razoável grau de informatização disponível no país (MS, 1998b). As maiores dificuldades diziam respeito, especialmente, às subnotificações dos agravos e à precariedade de informações sobre morbidade em âmbito nacional.

O SINAN foi concebido e desenvolvido pelo Centro Nacional de Epidemiologia (CENEPI), com o apoio técnico do Departamento de Informática do SUS (DATASUS) e do PRODABEL (Processamento de Dados do Município). Este tem por objetivo subsidiar a coleta e o processamento de dados sobre agravos de notificação em todo o território nacional, fornecendo informações para a análise do perfil de morbidade e contribuindo, desta forma, para a tomada de decisões em nível Municipal, Estadual e Federal (MS, 1998b).

Desde 1986, ano em que a AIDS passa a ser incluída como doença de notificação compulsória (MS, 1986), os casos de AIDS confirmados vinham sendo notificados regularmente. A implantação do SINAN/AIDS em 1993 significou um importante avanço, na medida em que favoreceu a uniformização dos bancos de dados e as análises epidemiológicas sobre AIDS no país. E, de fato, o sistema veio a propiciar avanços no sentido da racionalidade e capacidade local de gerenciamento dos dados de morbidade desta doença, como também sugeriu algumas linhas de investigação no campo do conhecimento e da intervenção em AIDS. Por outro lado, muitos foram, e têm sido, os embates técnico-operacionais no gerenciamento deste sistema, uma vez que foi criado para dar conta do registro de agravos de notificação compulsória e investigação epidemiológica de 41 tipos de agravos (MS, 1997).

Do ponto de vista histórico, Santos (1999) chama atenção, em relação às particularidades das notificações brasileiras, para o pioneirismo das iniciativas estaduais (no caso de São Paulo) e municipais (no caso do Rio de Janeiro) na formulação dos instrumentos utilizados pela vigilância epidemiológica, ainda que a autora destaque que o Município do Rio de Janeiro tenha estruturado sua atividade de vigilância fundamentando-se na experiência paulista até a estruturação do programa de AIDS do estado.

O gerenciamento dos dados de notificação e investigação de casos de AIDS do Município do Rio de Janeiro, desde 1986, passaram a ser de total responsabilidade do Programa de DST/ AIDS do Estado do Rio de Janeiro. Até 1993, o Estado cuidava não só da vigilância epidemiológica, mas era também de sua responsabilidade o gerenciamento da prevenção, assistência, repasse de medicamentos do Município do Rio, que, por sua vez, desde o início da epidemia, sempre teve uma importância singular no quantitativo de casos acumulados do Estado.

A partir de então, o gerenciamento do sistema de notificação foi repassado para a vigilância epidemiológica com a Coordenação de Programas de Epidemiologia da SMS-RJ. Este só foi assumido definitivamente pelo Programa de DST/AIDS em 1995, quando o mesmo passa a ser inserido na estrutura da Coordenação de Doenças Transmissíveis da SMS-RJ. 
A estruturação do Programa permitiu, todavia, que o Município se encarregasse do que lhe era de responsabilidade, dada a sua magnitude, potencial de disseminação e relevância social, em termos de casos acumulados de AIDS que somam 19.485 casos (DATASUS, 2000), assim como em termos de controle de recursos técnicos e financeiros. Este quantitativo de casos do Município do Rio de Janeiro representa um percentual de $65,5 \%$ do total de casos do Estado.

\section{A notificação de casos e o fluxo de dados de AIDS}

Num esforço de definição das diretrizes para o Programa de DST/AIDS do Município do Rio de Janeiro, um grupo de técnicos dos Programas de DST/AIDS do Município e Estado, coordenadores das áreas programáticas (AP) e representantes das unidades situadas em cada AP formularam normas e rotinas a serem implementadas pelo Programa. Dentre elas estava preconizado que, para fins de vigilância no nível local, a "notificação e investigação de casos confirmados de AIDS é compulsória, devendo ser registrada em formulário próprio e encaminhada ao nível central" (SMS-RJ, 1994:11).

Todas as unidades de saúde, independente de seu nível de complexidade, notificam casos de AIDS de adultos ou crianças, contanto que não deixem de cumprir as exigências preestabelecidas para a definição de caso. De acordo com Aguiar \& Fonseca (1998), para que o serviço de saúde notifique um caso, basta que ele: (a) identifique os "casos fechados" de AIDS; (b) realize o preenchimento correto da ficha de notificação/investigação epidemiológica de AIDS; (c) encaminhe para o órgão central do programa de AIDS do município.

Considerando, então, os diferentes níveis de atuação do programa e de suas competências, foi estabelecido no nível nacional o seguinte fluxo de dados que compreendia desde a produção do dado até a divulgação das informações analisadas e consolidadas:

Importante destacar que, embora sigam o mesmo fluxo de informações, o sistema de vigilância de AIDS de criança e adulto se distinguem em vários aspectos. Ambos vêm organizando-se mediante as informações geradas pelo sistema de informação do SINAN, cuja fonte básica de informação é a ficha individual de notificação/investigação dos casos confirmados de AIDS em criança ou em adulto. Após notificação/investigação do caso confirmado, segundo o fluxo de dados preestabelecido, as fichas são encaminhadas para digitação e críti- cas na SMS e, posteriormente, enviadas à Secretaria Estadual de Saúde (SES) e Programa Nacional de DST/AIDS (MS, 1998b), segundo demonstrado no fluxo acima.

Existem dois bancos de notificação gerenciados pelo Programa de DST/AIDS: um que compreende a notificação dos casos de HIV/ AIDS de indivíduos com 13 anos de idade ou mais, conforme os requisitos estabelecidos pela Definição Nacional de Casos de AIDS em Adultos (MS, 1998a); e outro que compreende os menores de 13 anos de idade e Definição de Caso de AIDS em Crianças (MS, 2000). E o que basicamente distingue um do outro é a diferença nas definições de casos, que incluem diferentes itens nos critérios de definição para que sejam incluídos no banco de dados.

A Tabela 1 procura sintetizar os critérios definidores de casos, desde a publicação da primeira definição de caso feita em 1982 pelo Centers for Disease Control and Prevention (CDC).

Diferente então de outros agravos, a AIDS possui um único formulário de entrada no SINAN, que contempla a Ficha Individual de Notificação (FIN) e a Ficha Individual de Investigação (FII), haja vista a necessidade de o indivíduo ser notificado somente por meio dos critérios estabelecidos para a caracterização de caso de AIDS. As principais fontes de informação para o sistema de vigilância de AIDS são: os serviços de assistência médica ambulatorial e hospitalar de referência; os consultórios e hospitais da rede privada além dos serviços de estatística de mortalidade (Sanches, 1998).

Desde o início da notificação de casos no Município do Rio de Janeiro, em 1982, foram utilizados diferentes modelos de ficha de notificação e investigação de AIDS. De acordo com estudo desenvolvido por Santos (1999), foram um total de quinze modelos diferenciados de notificação/investigação de casos de AIDS, no período de 1982 até 1998. Tais mudanças refletem a necessidade de se dar conta das várias revisões de caso de AIDS, conforme mencionado acima, buscando-se adequá-las aos novos conhecimentos que foram incorporados sobre a história natural da infecção.

De qualquer forma, o que se pode observar é que tanto as alterações nas fichas de notificação/investigação de AIDS quanto nas versões do SINAN remetem-nos ao raciocínio desenvolvido por Carneiro Jr. (1995), quando o mesmo faz considerações apontando que a história natural da infecção pelo HIV e o processo epidêmico da AIDS vêm demandando esforços de investigação e reformulações de conceitos e técnicas na perspectiva da vigilância epidemiológica deste agravo. Tal constatação evoca 
o quanto que a epidemia de AIDS trouxe, para o campo do conhecimento e das práticas de saúde, sobremodo para as ações de vigilância epidemiológica, questões desconhecidas e que não se enquadravam nos modelos clássicos de controle das doenças transmissíveis, como: a questão comportamental, as técnicas sofisticadas da imunologia, etc.

Buchalla (1995), ao fazer uma crítica ao sistema de vigilância de AIDS, considerou que este possui duas fontes de erro que são básicas: a não-identificação do indivíduo como caso de AIDS, pelo desconhecimento da definição, e a não-notificação do caso no sistema. Ao enfatizar esses erros, a autora levanta uma controvérsia presente na não-disponibilização do sistema para a notificação dos infectados assintomáticos, que não são aceitos, à medida que não fecham caso, segundo os critérios estabelecidos. Mas como ficam esses "não casos” para efeito da análise de difusão da epidemia e do planejamento de ações?

Com base nessa idéia, Carneiro Jr. (1995) destaca a construção de uma vigilância epidemiológica que se caracterizou como um sistema de informação sobre casos confirmados de AIDS, não conseguindo englobar os soropositivos assintomáticos, e nem dando conta desse segmento competente da epidemia. Ou seja, não consegue, assim, cumprir o seu papel de controle eficiente da epidemia, já que os soropositivos sintomáticos ou assintomáticos são tratados e não notificados.

$\mathrm{O}$ atendimento clínico e os resultados diagnósticos das pessoas infectadas pelo HIV geram notificações não reconhecidas como caso e descartadas pelo sistema. Isto é, a recomendação é a de que o serviço deve acompanhar os soropositivos sintomáticos ou não até que estes venham fechar caso; só então enviar a ficha para o nível central. Contudo, é uma prática corrente o envio de fichas que não fecham caso, que posteriormente são devolvidas à unidade de saúde. Isso demonstra a dificuldade na compreensão do fechamento do caso, assim como a predominância da lógica de notificação de casos suspeitos ou de confirmação laboratorial.

Sendo assim, é devidamente paradoxal compreender a não inclusão de um indivíduo como caso, mesmo sendo ele um portador do vírus, sintomático ou não. Mas isso corresponde a uma lógica diferenciada de compreensão que não favorece, particularmente, o controle mais precoce e efetivo da epidemia. Neste caso, observa-se que a vigilância de AIDS, ao priorizar os casos conhecidos e informados consoante os critérios de definição de caso e ao estruturar

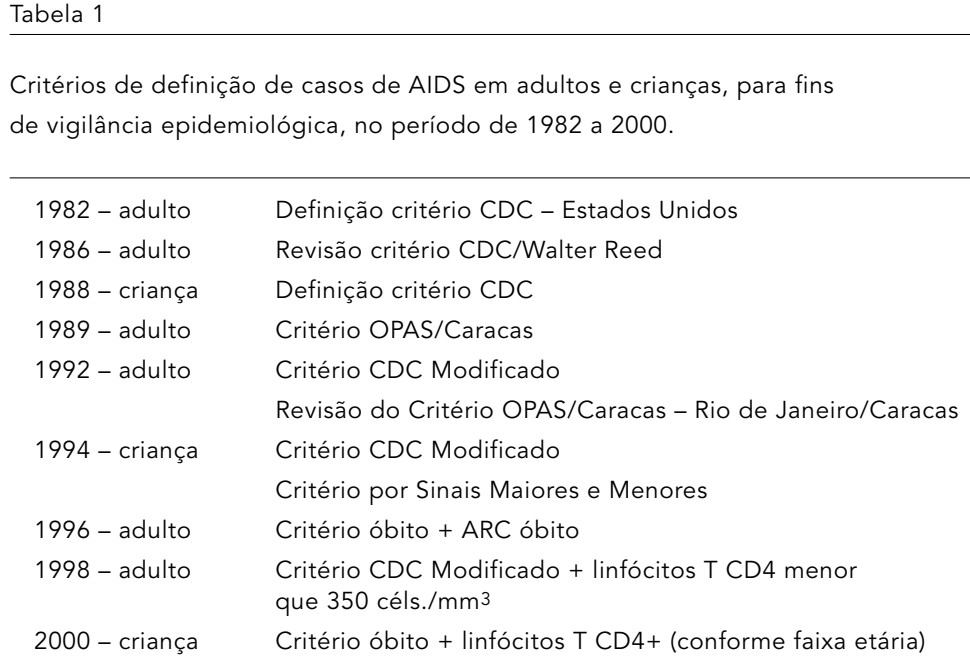

Fonte: adaptado da SES-RJ (1998).

$C D C=$ Centers for Disease Control and Prevention; OPAS = Organização

Pan-Americana da Saúde; ARC = AIDS Related Complex.

indicadores com base nesses dados, não favorece o desenvolvimento de ações de redução do impacto da epidemia, devido a esse significativo atraso. Isso evidencia, conforme mencionado anteriormente, a necessidade de investimento na incorporação das distintas dimensões da vigilância do HIV de segunda geração.

\section{Potencialidades do Sistema de Informação Nacional de Agravos de Notificação/AIDS como instrumento da vigilância}

Para melhor destacar os pontos a serem discutidos em termos de qualidade do sistema e ao considerar a análise das variáveis com maior índice de perdas dos dados de notificação de HIV/AIDS do SINAN, são apresentadas como resultado a etapa de trabalho (que correspondeu ao levantamento de todas as fichas com dados ignorados e sem informação para o período de 1985 a 1995), a atualização da base de dados após compatibilização e recuperação das informações das diversas fichas existentes e analisadas e a devolução atualizada do banco de dados para a Gerência do Programa de DST/ AIDS do Município do Rio de Janeiro (Cruz, 1999).

Do total de 11.318 fichas de notificação selecionadas para o período de 1985 a 1995, foram atualizadas 5.704 fichas que estavam com pelo menos um dado ignorado ou sem informação (fichas incompletas), sendo 5.403 fichas de adultos (indivíduos com 13 anos ou mais) e 301 fichas de crianças (indivíduos com menos de 13 anos). Desse total não foi possível encontrar 46 fichas de adultos e 66 fichas de crianças. 
As variáveis identificadas como problemáticas e selecionadas para serem atualizadas foram: bairro de residência, categoria de exposição, escolaridade e ocupação. Estas foram selecionadas não só por serem as que apresentavam um maior percentual de perdas, como também, pelo fato de serem variáveis epidemiológicas de grande valor na explicação da dinâmica da epidemia no Município. A atualização se deu por intermédio da recuperação de informações nas fichas com posterior preenchimento dos campos na base de dados.

Um outro problema diz respeito à ausência de dados que seriam fundamentais do ponto de vista do entendimento do caráter dinâmico da epidemia ou de seus possíveis fluxos, como, por exemplo, o endereço do local de trabalho, um campo especial que considere quem vive na rua; e, especialmente, algum item que investigue a prevenção.

$\mathrm{O}$ fato desses dados serem colocados em segundo plano sugere um interesse maior sobre o conhecimento clínico da doença, em detrimento de informações que compõem o processo de investigação. Pensando no aprimoramento do sistema de vigilância, temos obrigatoriamente de chamar a atenção para tais precariedades.

No levantamento realizado, foi observada a grande freqüência de fichas que tiveram dados incluídos ou alterados em razão da investigação feita por meio de prontuários de pacientes; notificação de um mesmo paciente feita algumas vezes em até seis diferentes modelos de ficha ou mesmo anotações complementares na própria ficha ou em qualquer outro papel anexado a ela.

\section{Limitações do Sistema de Informação Nacional de Agravos de Notificação/AIDS como instrumento da vigilância}

O levantamento realizado demonstrou a necessidade de uma maior compreensão do que é denominado "informação ignorada”, uma vez que a mesma foi percebida como possuindo diferentes tipos de interpretações. Em determinadas situações, a informação ignorada significava um dado ignorado por parte do informante; uma resposta sem informação, ou mesmo uma resposta que, apesar de preenchida, não era aceita pelo sistema e passava a ser identificada como ignorada.

Tal apreciação se torna pertinente ao passo que se constata um número considerável de preenchimento de informações ignoradas, que, na realidade, correspondem, em sua maioria, a campos sem informação, como por exemplo para as variáveis escolaridade e ocupação. Outro exemplo capaz de retratar a ambigüidade do significado da informação ignorada diz respeito ao preenchimento da categoria de exposição. O campo ignorado para categoria de exposição estava referido a campo sem preenchimento, a campo ignorado (campo 9), ou, no caso de categoria heterossexual sem menção ao comportamento de risco do informante ou de seu parceiro.

No processo de atualização das fichas, foi observado que o sistema foi programado para reconhecer os heterossexuais sem fator de risco associado com ignorado, o que representa uma incongruência. Primeiro, por elevar o quantitativo de ignorados que na realidade não é ignorado, certamente subestimando o número de casos de transmissão heterossexual; segundo, por ser esta uma das principais formas de transmissão do HIV/AIDS no país, em especial do sexo feminino. Isso sem levar em conta as perdas que se dão em função de erro de classificação da categoria de exposição, em virtude das características da cultura sexual brasileira, consoante mencionam Daniel \& Parker (1991).

Essa distorção nos leva a pensar: quem de fato são esses heterossexuais ignorados pelo sistema? Por que ao invés de ignorá-los não se criou um sistema mais eficiente para serem investigados os casos heterossexuais que se informam? De alguma forma esses dados ignorados apontam para uma exigência de revisão das fichas com categorias de exposição ignorada, ou mesmo de reformulação do próprio sistema nesse tópico.

Um dos aspectos observados em relação à variável bairro de residência é que esta informação aparece freqüentemente condicionada pela questão do acesso ao atendimento. Não se descartou também a aparição de bairros de residência condizentes ao bairro de localização da ocupação ou bairro de residência substituído pelo bairro da instituição a que o sujeito encontrava-se confinado ou sendo tratado. Dos diferentes modelos de ficha manuseados, foi identificado apenas um modelo bem antigo que possuía o campo endereço do trabalho.

Evidenciou-se também como fundamental a inclusão, na caracterização da epidemia na cidade, de informações sobre os registros de "moradores de rua". Afinal, não era sequer possível, pelas notificações, saber os bairros em que essas pessoas moravam. Em geral o campo referente a local de residência dos moradores de rua vinham totalmente em branco, e na atualização apenas acrescentou-se em referência para o endereço a terminologia "população de rua". 
Ligada ainda à questão dos moradores de rua, aparece com grande freqüência, como ocupação, os mendigos e catadores de papel. $\mathrm{Na}$ maioria das vezes esses campos ficam sem preenchimento ou são digitados como "outros”, visto a não-aceitação destas ocupações, que não se enquadram como formais. É importante apenas destacar que esta denota uma significativa mudança estrutural na condição sócio-econômica da população com AIDS, e, por outro lado, pela sua mobilidade integram diferentes circuitos das metrópoles, podendo viabilizar a disseminação da doença (Sabroza et al., 1995).

De todas as variáveis analisadas, a variável ocupação foi considerada uma das mais problemáticas em todos os aspectos, refletida no percentual de perdas dessa variável. É perceptível a dificuldade na decodificação da variável ocupação. Para ilustrar tal fato, pode-se observar que a informação doméstica ou do lar tem as seguintes opções no sistema: doméstica (empregada), doméstica (dona-de-casa), dona-decasa e empregada doméstica. Mas como diferenciá-las? As interpretações dadas são as mais diversas possíveis, o que complica o momento da especificação.

Para chamar a atenção de alguns pontos interessantes, verificou-se que uma das ocupações que apareceu com bastante freqüência foi a de "profissional do sexo", que não tinha nenhuma possibilidade de codificação (tabela adotada do Código Brasileiro de Ocupações) pelo sistema, por mais que saibamos da importância desta informação, em particular na questão do fator de exposição à infecção ao HIV. Todas as fichas que constavam "profissional do sexo" ou qualquer outra denominação semelhante eram registradas como sem informação, e foi mantida assim na atualização de dados em razão da limitação do sistema que não aceita a atividade como um tipo de ocupação.

Dos dados ignorados e sem informação de casos notificados de AIDS no período de 1985 a 1995, antes da atualização, foram verificados os seguintes índices de perda (Tabela 2), dos 11.016 casos de adultos notificados:

A distribuição proporcional mostrou uma alteração estatisticamente significativa após atualização feita das variáveis bairro e ocupação que apresentaram, respectivamente, um $\chi^{2}=29,36$ e 19,11 ( $\left.p=0,000\right)$. Enquanto que, em relação às demais variáveis não houve uma diferença estatisticamente significante, principalmente do valor encontrado para a variável escolaridade que teve um $\chi^{2}=0,006$ ( $\left.p>0,005\right)$.

Mesmo não tendo a intenção de comparar o conteúdo de tais variáveis, mas apenas dan-
Tabela 2

Percentual de dados ignorados antes e depois da atualização da base de dados do Município do Rio de Janeiro, 1985-1995.

\begin{tabular}{lcc}
\hline Variáveis & Antes (\%) & Depois (\%) \\
\hline Bairro & 48,2 & 12,9 \\
Ocupação & 50,4 & 20,8 \\
Escolaridade & 27,9 & 27,7 \\
Categoria de exposição & 25,9 & 24,0
\end{tabular}

Fonte: SMS-RJ (1999).

do ênfase aos campos ignorados, observa-se que tais evidências sugerem que as perdas nas variáveis bairros e ocupação podem estar mais relacionadas a problemas de inconsistências do próprio sistema. Já o ocorrido com as variáreis escolaridade e categoria de exposição parece envolver mais problemas referentes à própria abordagem da investigação feita, no momento anterior de preenchimento da ficha.

Um outro problema-chave a ser destacado é a não possibilidade de intercâmbio do SINAN com outros sistemas de informação, como por exemplo SIM (Sistema de Informação de Mortalidade), a AIH (Sistema de Autorização de Internação Hospitalar) e o SICLOM (Sistema de Informação de Controle Logístico de Medicamentos), entre outros, por não existirem padrões, cadastros ou tabelas compatíveis (MS, 1997) que permitiriam a compatibilização dos sistemas. Essa falta de articulação só dificulta o processo de vigilância à proporção que se subtilizam informações de morbidade, mortalidade, sócio-econômicas e demográficas geradas por outros sistemas.

O cruzamento do SINAN com o SIM pode interferir diretamente na subnotificação de casos de AIDS, já que o óbito por esta causa é um dos critérios de definição de caso. O outro exemplo é o cruzamento do SINAN com o SICLOM para a identificação de soropositivos que fecham caso segundo critério linfócito T CD4 abaixo de 350 céls $/ \mathrm{mm}^{3}$.

Ferreira \& Portela (1999) ressaltam o quanto a subnotificação de casos de AIDS pode implicar uma estimativa equivocada da magnitude e ônus da epidemia, acarretando uma subalocação de ações e recursos para o seu enfrentamento. Ao tratarem da subnotificação, as autoras se reportam ao desconhecimento do número real de casos de AIDS em menores de 13 anos de idade, o que, por sua vez, prejudica tanto a normatização da epidemia, quanto a avaliação das ações preventivas, inviabilizando 
Figura 1

Fluxo de notificação de casos de AIDS.

Nível local (Unidade de Saúde)

Responsável pelo atendimento, produção dos dados

e investigação epidemiológica.

regional (Vigilância Epidemiológica da SMS)

É o nível intermediário entre a Coordenação Estadual e o nível municipal.

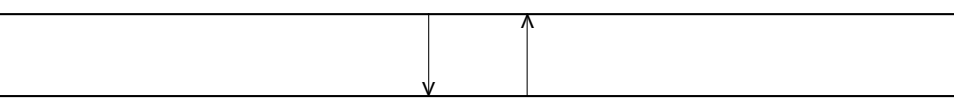

Nível estadual (Assessoria de DST/AIDS da SES)

Responsável pela revisão das investigações realizadas, organização

e consolidação dos dados e envio para o PN DST/AIDS.

Responsável pela revisão dos registros, processamento e análise dos dados

e elaboração do Boletim epidemiológico para retroalimentação dos sistema.

SMS = Secretaria Municipal de Saúde; SES = Secretaria Estadual de Saúde; PN DST/AIDS = Programa Nacional de DST e AIDS; CN DST/AIDS = Coordenação Nacional de DST e AIDS ções técnicas e operacionais, inerentes ao SINAN, inviabilizam a construção de um diagnóstico mais próximo das características epidemiológicas da epidemia do HIV/AIDS na cidade, considerando-se a sua dimensão mais ampla e dinâmica.

De um modo geral, notou-se que a lógica do sistema de vigilância de HIV/AIDS, como a de outros sistemas da área da saúde, está centralizada na racionalidade biomédica, enfatizando de forma mais contundente o caráter assistencial do que o preventivo. Isso requer um aprimoramento do sistema com objetivos claros e direcionados ao subsídio de informações à intervenção nos diferentes níveis de prevenção e de controle da epidemia de AIDS. Talvez fosse coerente a presença, por exemplo, de um campo específico, na ficha de notificação/investigação, para a abordagem do acesso ao preservativo, ou mesmo, ao seu uso propriamente dito.

Observa-se que a lógica de vigilância do HIV/AIDS não se diferencia da lógica de vigilância epidemiológica dos demais tipos de agravos, na forma de serem concebidos e na estruturação do sistema. Isto denota restrições de ordem conceitual e operacionais que podem estar remetidos a vieses ideológicos condizentes à vinculação a uma estrutura rígida e hierarquizada de vigilância epidemiológica já consolidada.

Com uma estrutura devidamente pautada nos parâmetros estabelecidos pela definição de caso e não-caso de AIDS, entende-se que o sistema de vigilância foi construído com base numa concepção fundada na doença, vale dizer, o controle da transmissão é colocado no mesmo patamar do manejo de caso. Esta equivalência contribui para a prevenção. Dessa forma a investigação de outros fatores - que possam vir a orientar a adoção de medidas de prevenção mais adequadas - pode estar comprometida pela não-inclusão de todos os infectados pelo HIV. Neste sentido, a primazia da notificação segundo critérios de definição de caso e a estrutura de indicadores com base nesses dados, já provou não favorecer o desenvolvimento e o acompanhamento de ações para a redução do impacto da epidemia.

De um modo geral, as considerações feitas visam salientar a importância de se tratar o SINAN/AIDS como um instrumento de vigilância, reconhecendo todas as suas potencialidades e as possibilidades de intervenção no aprimoramento da qualidade dos dados e da confiabilidade do sistema. Pensar em intervir na qualidade do sistema de vigilância de AIDS, e, conseqüentemente, em seu sistema de informação, significa investir na capacitação de téc- 
nicos em vigilância para desencadear processos de investigação, no estreitamento da distância entre formação profissional e conceito-princípios do Sistema Nacional de Vigilância Epidemiológica (SNVE) e a incorporação dos preceitos do SNVE no SINAN em todos os níveis.

Aprimorar o sistema de vigilância de AIDS no Município do Rio de Janeiro, neste sentido, implicaria um investimento que envolve capacitação de recursos humanos, ampliação de suporte técnico, descentralização do sistema, construção de um sistema de crítica mais criterioso, uma melhor utilização e divulgação das informações produzidas. É, acima de tudo, valorizar a mudança da cultura institucional no que concerne à informação enquanto enfrentamento da epidemia na cidade.

\section{Referências}

AGUIAR, A. C. \& FONSECA, A. F., 1998. Vigilância epidemiológica da AIDS. In: Textos de Apoio em Vigilância Epidemiológica (Escola Politécnica de Saúde Joaquim Venância, org.), Série Trabalho e Formação em Saúde, pp. 85-89, Rio de Janeiro: Editora Fiocruz.

BUCHALLA, C. M., 1995. AIDS: O surgimento e a evolução da doença. In: Velhos e Novos Males da Saúde no Brasil: A Evolução do País e de suas Doenças (C. A. Monteiro, org.), pp. 331-356, São Paulo: Editora Hucitec.

CARNEIRO Jr., N., 1995. Vigilância Epidemiológica de AIDS no Município de Santo André - Um Sistema de Informação para o Controle: Um Estudo de suas Características. Dissertação de Mestrado, São Paulo: Departamento de Medicina Preventiva, Faculdade de Medicina, Universidade de São Paulo.

CRUZ, M. M., 1999. A Epidemia de HIVIAIDS na Cidade do Rio de Janeiro e seus Padrões de Espacialida$d e$. Dissertação de Mestrado, Rio de Janeiro: Escola Nacional Saúde Pública, Fundação Oswaldo Cruz.

DANIEL, H. \& PARKER, R., 1991. AIDS - A Terceira Epidemia: Ensaios e Tentativas. São Paulo: Iglu.

DATASUS (Departamento de Informática do SUS), 2000. Sistema de Informação Nacional de Agravos de Notificação. 23 Agosto $2000<$ http// www.datasus. gov.br>.

FERREIRA, V. M. \& PORTELA, M. C., 1999. Avaliação da subnotificação de casos de AIDS no Município do Rio de Janeiro com base em dados do sistema de informações hospitalares do Sistema Único de Saúde. Cadernos de Saúde Pública, 15:317-324.

MS (Ministério da Saúde), 1986. Portaria no 542 de 22 de dezembro de 1986. Brasília: Diário Oficial da União, p. 19827, 24 dez. Seção I.

MS (Ministério da Saúde), 1997. Compatibilização de Sistemas e Base de Dados (CBD) da Rede Integrada de Informação para a Saúde (RIPSA). Informe Epidemiológico do SUS, 6:35-41.

MS (Ministério da Saúde), 1998a. Revisão da Definição Nacional de Caso de AIDS em Indivíduos com 13 Anos de Idade ou Mais, para Fins de Vigilância Epidemiológica. Brasília: Coordenação de DST/
Enfim, a perspectiva é a de aproximação da construção de sistema(s) de informação capaz(es) de subsidiar a análise de processos complexos, como os que envolvem o processo de infecção pelo HIV, considerando-se diferentes práticas sexuais, estilos de vida, diferentes acessos a meios de prevenção e assistência, ou mesmo, diferentes vulnerabilidades. Essas são questões de tamanha relevância quando se vislumbra atuar, em termos de vigilância à saúde, no caminho do monitoramento e da avaliação das ações planejadas e executadas no controle do HIV/AIDS. Por fim, sem dúvida, a proposta de vigilância do HIV de segunda geração virá melhorar nossa capacidade de construção de um novo modelo de vigilância mais ampliada do HIV/AIDS.

AIDS, Secretaria de Projetos Especiais de Saúde.

MS (Ministério da Saúde), 1998b. Guia de Vigilância Epidemiológica. Brasília: Fundação Nacional de Saúde.

MS (Ministério da Saúde), 1999. A Resposta Brasileira ao HIVIAIDS: Experiências Exemplares. Brasília: Coordenação Nacional de DST/AIDS, Secretaria de Políticas de Saúde.

MS (Ministério da Saúde), 2000. Revisão da Definição Nacional de Caso de AIDS em Indivíduos Menores de 13 Anos, para Fins de Vigilância Epidemiológica. Brasília: Secretaria de Projetos Especiais de Saúde, Coordenação Nacional de DST/AIDS.

SABROZA, P. C.; KAWA, H. \& CAMPOS, W. S. Q., 1995. Doenças transmissíveis: Ainda um desafio. In: Os Muitos Brasis: Saúde e População na Década de 80 (M. C. S. Minayo, org.), pp. 177-244, Rio de Janeiro: ABRASCO.

SANTOS, A. L. G., 1999. Uma Construção dos Saberes sobre a Epidemia de AIDS - Os Formulários de Notificação de Casos em Perspectiva (1982-98). Dissertação de Mestrado, Rio de Janeiro: Escola Nacional de Saúde Pública, Fundação Oswaldo Cruz.

SANCHES, K. R. B., 1998. Sistema de Informação em HIVIAIDS - Manual de Vigilância Epidemiológica. Rio de Janeiro: Assessoria de DST e AIDS, Superintendência de Saúde Coletiva, Secretaria de Estado de Saúde do Rio de Janeiro.

SMS-RJ (Secretaria Municipal de Saúde do Rio de Janeiro), 1994. Manual de Normas e Rotinas do Programa de Doenças Sexualmente Transmissíveis e AIDS. Rio de Janeiro: Superintendência de Saúde Coletiva, Coordenação de Doenças Transmissíveis, Gerência de DST e AIDS.

UNAIDS (Joint United Nations Programme on HIV/ AIDS)/WHO (World Health Organization), 2000. Guidelines for Second Generation HIV Surveillance: The Next Decade. 23 September 2001 <http://www. unaids.org>.

Recebido em 10 de julho de 2001

Versão final reapresentada em 2 de outubro de 2001

Aprovado em 1 de julho de 2002 